\title{
On complementary quadratic fractional programming problem
}

\author{
Basiya K. Abdulrahim \\ Department of Mathematics, Faculty of Education, School of Science Education, University of Garmian, Kurdistan Region -Iraq \\ E-mail: Basiya2008@yahoo.com
}

Copyright $(\odot 2014$ Basiya K. Abdulrahim. This is an open access article distributed under the Creative Commons Attribution License, which permits unrestricted use, distribution, and reproduction in any medium, provided the original work is properly cited.

\begin{abstract}
In this paper, a class of optimization problems has been considered where quadratic fractional programming problem has an additional characteristic, i.e Complementary quadratic fractional programming problem (CQFPP) and consequently a convergent algorithm has been developed in the following discussion. Numerical examples have been provided in support of the theory. By using Matlab 2011 version 7.12.0.635 (R2011a).
\end{abstract}

Keywords: CQFPP, Solving CQFPP by new technique to modified simplex method.

\section{Introduction}

In (1969-1971) Ibaraki ([5], [6]) studied complementary programming, and defined a new type of optimization problems, known as complementary programming problems. In (1982) Gupta and Sharma had developed an algorithm for solving a quadratic complementary programming problem with indefinite [4]. In (2009) Arora and Narang are studies a bilevel fractional programming problem with independent followers [2]. In (2011) Judice studies algorithms for linear programming with linear complementarity constraints [8]. In (2010) Jahan and Islam are studied a complementary slackness theorem for linear fractional programming problem [7]. In (1989) Sulaiman [11] studied to quadratic complementary programming algorithms and their computer applications. In (2009) Fang, Gao, Sheu and Xing are studies to global optimization for class of fractional programming problems and quadratic fractional programming problem [3]. In (2011) Arora and Arora are studies to solving linear quadratic bilevel programming problem using Kuhn-Tucker conditions [1].

To extend this work, we have been defined CQFPP and investigated technique to generate the best compromising optimal solution.

\section{Complementary QFPP}

Ibaraki [6] defined complementary programming problem and Gupta and Sharma [4] defined complementary quadratic programming problem (CQPP), complementary quadratic fractional programming problem (CQFPP) can be defined as follows:

$\operatorname{Max} . Z=\frac{\left(c^{t} x+S^{t} u+s^{t} v+\gamma\right)\left(e^{t} x+O^{t} u+o^{t} v+\delta\right)}{\left(d^{t} x+W^{t} u+w^{t} v+\beta\right)\left(f^{t} x+G^{t} u+g^{t} v+\varepsilon\right)}$

Subject to:

$$
\begin{aligned}
& A x+B u+C v=b \\
& u v=0 \\
& x, u, v \geq 0
\end{aligned}
$$

Where $x, u, v$ are $n, m$-dimensional and $m$-dimensional vectors of variables respectively; $c, e, f$ and $d$ are $n$-dimensional and $S, O, W, G, S, o, w, g$ are $m$-dimensional vectors of constants respectively; $b$ is $p$-dimensional vector of constants; $A, B, C$ is $p \times n, p \times m$ and $p \times m$ matrix of constraints respectively, $\gamma, \delta, \beta, \varepsilon$ are constants 


\section{Modified simplex method development}

Simplex method is developed by Dantzig in (1947). The simplex method provides a systematic algorithm which consists of moving from one basic feasible solution (one vertex) to another in prescribed manner such that the value of the objective function is improved. This procedure of jumping from vertex to vertex is repeated. If the objective function is improved at each jump, then no basis can ever be repeated and there is no need to go back to vertex already covered. Since the number of vertices is finite, the process must lead to the optimal vertex in a finite number of steps. The simplex algorithm is an iterative (step by step) procedure for solving linear programming problems. It consists of:

i) Having a trail basic feasible solution to constraint equations.

ii) Testing whether is an optimal solution.

iii) Improving the first trial solution by a set of rules, and repeating the process till an optimal solution is obtained.

For more details [9]. Modified simplex method to solve linear fractional programming problem and to solve produced to linear programming problem [10]. Using new technique to modified simplex method to solve the numerical example to apply simplex process [10]. First we find $\Delta_{j 1}, \Delta_{j 2}, \Delta_{j 3}$ and $\Delta_{j 4}$ from the coefficients of numerator and denominator of objective function respectively, by using the following formula:

$\Delta_{j i}=C_{j i}-C_{B i} x_{j i}, i=1,2,3,4, j=1,2, \ldots, m+n$,

$z_{1}=C_{B 1} V_{B}+\gamma, z_{2}=C_{B 2} V_{B}+\delta, z_{3}=C_{B 3} V_{B}+\beta, z_{4}=C_{B 4} V_{B}+\varepsilon$,

$\gamma, \delta, \beta, \varepsilon$ are constants $, Z_{1}=z_{1} z_{2}, Z_{2}=z_{3} z_{4}, \quad Z=\frac{z_{1}}{z_{2}}$

$\mu_{j 1}=\mu_{j 2}=\min \left[\frac{V_{B}}{x_{j}}, x_{j}>0\right]$ for non - basic variables

$\Delta_{j a}=z_{1} \Delta_{j 2}+z_{2} \Delta_{j 1}+\mu_{j 1} \Delta_{j 1} \Delta_{j 2}, \Delta_{j b}=z_{3} \Delta_{j 4}+z_{4} \Delta_{j 3}+\mu_{j 2} \Delta_{j 3} \Delta_{j 4}$

In this approach we define the formula to find $\Delta_{j}$ from $Z_{1}, Z_{2}, \Delta_{j a}$ and $\Delta_{j b}$ as follows: $\Delta_{j}=Z_{2} \Delta_{j a}-Z_{1} \Delta_{j b}$. Here $C_{j i}$ are the coefficients of the basic and non-basic variables in the objective function and , $C_{B i}$ are the coefficients of the basic variables in the objective function, $j=1,2, \ldots, m+n, i=1,2,3,4$

For testing optimality solution must be all $\Delta_{j} \leq 0$ but here all $\Delta_{j}$ not lesser than zero, and then the solution is not optimal. Repeat the same approach to find next feasible solution.

\section{Algorithm for CQFPP}

The following algorithm is to obtain the optimal solution for the CQFPP by new technique to modified simplex method which can be summarized as follows:

Step1: Write the standard form of the problem without complementary condition $u v=0$, by introducing slack and artificial variables to constraints, and write starting new technique to modified simplex table.

Step2: Calculate the $\Delta_{j}$ by the following formula $\Delta_{j}=Z_{2} \Delta_{j a}-Z_{1} \Delta_{j b}$, then write it in the starting new technique to modified simplex table.

Step3: Find the solution by using new technique to modified simplex process without complementary condition $u v=$ 0 , examine whether the complementary condition holds or not.

Step4: If the complementary condition $u v=0$, then go to step7 otherwise go to step5.

Step5: If the complementary condition $u v=0$, is not satisfied use branch and bounded algorithm proposed by Ibaraki [6] modified by Gupta and Sharma [4] for quadratic objective function, and ensure that $u v=0$, go to step6.

Step6: Check the solution for feasibility in step3, if it is feasible then go to step7, otherwise use dual new technique to modified simplex method to remove infeasibility.

Step7: Check solution for optimality if all $\Delta_{j} \leq 0$, then the solution is optimal, otherwise go to step3.

\section{Numerical examples and results}

In this section we have presented only two examples among several examples that we have solved, to show the accuracy of the new technique and which new technique to modified simplex method is better and more convenience.

Example 5.1: We consider the following CQFPP as 
$\operatorname{Max} . Z=\frac{\left(4 x_{1}+2 x_{2}\right)\left(2 x_{1}+x_{2}\right)}{\left(x_{1}+x_{2}+2\right)\left(2 x_{1}+x_{2}+4\right)}$

Subject to:

$$
\begin{aligned}
& x_{1}+x_{2} \leq 6 \\
& 8 x_{1}+4 x_{2} \leq 32 \\
& x_{1} \leq 3 \\
& x_{2} \leq 5 \\
& x_{1} x_{2}=0 \\
& x_{1}, x_{2} \geq 0
\end{aligned}
$$

\begin{tabular}{|c|c|c|c|c|c|c|c|c|c|c|c|c|}
\hline & & & & & $\begin{array}{c}C_{j 1} \\
C_{j 2} \\
C_{j 3} \\
C_{j 4}\end{array}$ & $\begin{array}{l}4 \\
2 \\
1 \\
2\end{array}$ & $\begin{array}{l}2 \\
1 \\
1 \\
1\end{array}$ & $\begin{array}{l}0 \\
0 \\
0 \\
0\end{array}$ & $\begin{array}{l}0 \\
0 \\
0 \\
0\end{array}$ & $\begin{array}{l}0 \\
0 \\
0 \\
0\end{array}$ & $\begin{array}{l}0 \\
0 \\
0 \\
0\end{array}$ & \\
\hline B.V. & $C_{B 1}$ & $C_{B 2}$ & $C_{B 3}$ & $C_{B 4}$ & $V_{B}$ & $x_{1}$ & $x_{2}$ & $x_{3}$ & $x_{4}$ & $x_{5}$ & $x_{6}$ & Min ratio \\
\hline$x_{3}$ & 0 & 0 & 0 & 0 & 6 & 1 & 1 & 1 & 0 & 0 & 0 & $6 / 1=6$ \\
\hline$x_{4}$ & 0 & 0 & 0 & 0 & 32 & 8 & 4 & 0 & 1 & 0 & 0 & $32 / 8=4$ \\
\hline$x_{5}$ & 0 & 0 & 0 & 0 & 3 & 1 & 0 & 0 & 0 & 1 & 0 & $3 / 1=3$ \\
\hline$x_{6}$ & 0 & 0 & 0 & 0 & 5 & 0 & 1 & 0 & 0 & 0 & 1 & - \\
\hline \multirow{3}{*}{\multicolumn{5}{|c|}{$\begin{array}{l}z_{1}=0 \\
z_{2}=0 \\
Z_{1}=z_{1} z_{2}=0\end{array}$}} & $\Delta_{j 1}$ & 4 & 2 & 0 & 0 & 0 & 0 & \\
\hline & & & & & $\Delta_{j 2}$ & 2 & 1 & 0 & 0 & 0 & 0 & \\
\hline & & & & & $\mu_{j 1}$ & 3 & 5 & 0 & 0 & 0 & 0 & \\
\hline \multirow{3}{*}{\multicolumn{5}{|c|}{$\begin{array}{l}z_{3}=2 \\
z_{4}=4 \\
Z_{2}=z_{3} z_{4}=8\end{array}$}} & $\overline{\Delta_{j 3}}$ & 1 & 1 & 0 & 0 & 0 & 0 & \\
\hline & & & & & $\Delta_{j 4}$ & 2 & 1 & 0 & 0 & 0 & 0 & \\
\hline & & & & & $\mu_{j 2}$ & 3 & 5 & 0 & 0 & 0 & 0 & \\
\hline \multirow{3}{*}{\multicolumn{5}{|c|}{$\begin{array}{l}Z_{1}=0 \\
Z_{2}=8 \\
Z=\frac{Z_{1}}{Z_{2}}=0\end{array}$}} & $\overline{\Delta_{j a}}$ & 24 & 10 & 0 & 0 & 0 & 0 & \\
\hline & & & & & $\Delta_{j b}$ & 14 & 11 & 0 & 0 & 0 & 0 & \\
\hline & & & & & $\Delta_{j}$ & 192 & 80 & 0 & 0 & 0 & 0 & \\
\hline
\end{tabular}

Solution 5.1: Solving the example 5.1 by new technique to modified simplex method and applied an algorithm in the

\begin{tabular}{|c|c|c|c|c|c|c|c|c|c|c|c|c|}
\hline & & & & & $\begin{array}{l}C_{j 1} \\
C_{j 2} \\
C_{j 3} \\
C_{j 4}\end{array}$ & $\begin{array}{l}4 \\
2 \\
1 \\
2\end{array}$ & $\begin{array}{l}2 \\
1 \\
1 \\
1\end{array}$ & $\begin{array}{l}0 \\
0 \\
0 \\
0\end{array}$ & $\begin{array}{l}0 \\
0 \\
0 \\
0\end{array}$ & $\begin{array}{l}0 \\
0 \\
0 \\
0\end{array}$ & $\begin{array}{l}0 \\
0 \\
0 \\
0\end{array}$ & \\
\hline B.V. & $C_{B 1}$ & $C_{B 2}$ & $C_{B 3}$ & $C_{B 4}$ & $V_{B}$ & $x_{1}$ & $x_{2}$ & $x_{3}$ & $x_{4}$ & $x_{5}$ & $x_{6}$ & Min ratio \\
\hline$x_{3}$ & 0 & 0 & 0 & 0 & 1 & 0 & 0 & 1 & $-1 / 4$ & 1 & 0 & \\
\hline$x_{2}$ & 2 & 1 & 1 & 1 & 2 & 0 & 1 & 0 & $1 / 4$ & -2 & 0 & \\
\hline$x_{1}$ & 4 & 2 & 1 & 2 & 3 & 1 & 0 & 0 & 0 & 1 & 0 & \\
\hline$x_{6}$ & 0 & 0 & 0 & 0 & 3 & 0 & 0 & 0 & $-1 / 4$ & 2 & 1 & \\
\hline$z_{1}=16$ & & & & & $\overline{\Delta_{j 1}}$ & 0 & 0 & 0 & $-2 / 4$ & 0 & 0 & \\
\hline$z_{2}=8$ & & & & & $\Delta_{j 2}$ & 0 & 0 & 0 & $-1 / 4$ & 0 & 0 & \\
\hline$Z_{1}=z_{1}$ & $=12$ & & & & $\mu_{j 1}$ & 0 & 0 & 0 & 8 & 1 & 0 & \\
\hline$z_{3}=7$ & & & & & $\Delta_{j 3}$ & 0 & 0 & 0 & $-1 / 4$ & 1 & 0 & \\
\hline$z_{4}=12$ & & & & & $\Delta_{j 4}$ & 0 & 0 & 0 & $-1 / 4$ & 0 & 0 & \\
\hline$Z_{2}=z_{3}$ & $=84$ & & & & $\mu_{j 2}$ & 0 & 0 & 0 & 8 & 1 & 0 & \\
\hline$\overline{Z_{1}}=12$ & & & & & $\overline{\Delta_{j a}}$ & 0 & 0 & 0 & -7 & 0 & 0 & \\
\hline$Z_{2}=84$ & & & & & $\Delta_{j b}$ & 0 & 0 & 0 & $-17 / 4$ & 12 & 0 & \\
\hline$Z=\frac{Z_{1}}{Z_{2}}$ & & & & & $\Delta_{j}$ & 0 & 0 & 0 & -44 & -1536 & 0 & \\
\hline
\end{tabular}
section 4, after 2 steps we obtained the initial table as follows in table 5.1.1, after two iterations, we obtained the result in the following table 5.1.2.

Table 5.1.1: Initial table for example 5.1 by new technique to modified simplex method

Table 5.1.2: Final table for example 5.1 by new technique to modified simplex method

After solving it by new technique to modified simplex method, without complementary condition $\left(x_{1} x_{2}=0\right)$ we get Max. $Z=\frac{128}{84}$ and $x_{1}=3, x_{2}=2$ 
Now, we going to satisfied the complementary condition $\left(x_{1} x_{2}=0\right)$, because $x_{1} x_{2} \neq 0$. The optimal solution is $\operatorname{Max} . Z=\frac{72}{50}$ and $x_{1}=3, x_{2}=0$

Example 5.2: We consider the following CQFPP as

$\operatorname{Max} . Z=\frac{\left(6 x_{1}+10 x_{2}+9 x_{3}\right)\left(x_{1}+x_{2}+x_{3}\right)}{\left(2 x_{1}+2 x_{2}+2 x_{3}+4\right)\left(x_{1}+x_{2}+x_{3}+5\right)}$

Subject to:

$$
\begin{aligned}
& x_{1}+3 x_{2}+2 x_{3} \leq 9 \\
& 3 x_{1}+2 x_{2}+x_{3} \leq 8 \\
& 2 x_{1}+x_{2}+3 x_{3} \leq 7 \\
& x_{2} x_{3}=0 \\
& x_{1}, x_{2}, x_{3} \geq 0
\end{aligned}
$$

\begin{tabular}{|c|c|c|c|c|c|c|c|c|c|c|c|c|}
\hline & & & & & $\begin{array}{c}C_{j 1} \\
C_{j 2} \\
C_{j 3} \\
C_{j 4}\end{array}$ & $\begin{array}{l}6 \\
1 \\
2 \\
1\end{array}$ & $\begin{array}{c}10 \\
1 \\
2 \\
1\end{array}$ & $\begin{array}{l}9 \\
1 \\
2 \\
1\end{array}$ & $\begin{array}{l}0 \\
0 \\
0 \\
0\end{array}$ & $\begin{array}{l}0 \\
0 \\
0 \\
0\end{array}$ & $\begin{array}{l}0 \\
0 \\
0 \\
0\end{array}$ & \\
\hline B.V. & $C_{B 1}$ & $C_{B 2}$ & $C_{B 3}$ & $C_{B 4}$ & $V_{B}$ & $x_{1}$ & $x_{2}$ & $x_{3}$ & $x_{4}$ & $x_{5}$ & $x_{6}$ & Min ratio \\
\hline$x_{4}$ & 0 & 0 & 0 & 0 & 9 & 1 & 3 & 2 & 1 & $\overline{0}$ & 0 & $9 / 3=3$ \\
\hline$x_{5}$ & 0 & 0 & 0 & 0 & 8 & 3 & 2 & 1 & 0 & 1 & 0 & $8 / 2=4$ \\
\hline$x_{6}$ & 0 & 0 & 0 & 0 & 7 & 2 & 1 & 3 & 0 & 0 & 1 & $7 / 1=7$ \\
\hline \multirow{3}{*}{\multicolumn{5}{|c|}{$\begin{array}{l}z_{1}=0 \\
z_{2}=0 \\
Z_{1}=z_{1} z_{2}=0\end{array}$}} & $\Delta_{j 1}$ & 6 & 10 & 9 & 0 & 0 & 0 & \\
\hline & & & & & $\Delta_{j 2}$ & 1 & 1 & 1 & 0 & 0 & 0 & \\
\hline & & & & & $\mu_{j 1}$ & $8 / 3$ & 3 & $7 / 3$ & 0 & 0 & 0 & \\
\hline \multirow{3}{*}{\multicolumn{5}{|c|}{$\begin{aligned} z_{3} & =4 \\
z_{4} & =5 \\
Z_{2} & =z_{3} z_{4}=20\end{aligned}$}} & $\Delta_{j 3}$ & 2 & 2 & 2 & 0 & 0 & 0 & \\
\hline & & & & & $\Delta_{j 4}$ & 1 & 1 & 1 & 0 & 0 & 0 & \\
\hline & & & & & $\mu_{j 2}$ & $8 / 3$ & 3 & $7 / 3$ & 0 & 0 & 0 & \\
\hline \multirow{3}{*}{\multicolumn{5}{|c|}{$\begin{array}{l}Z_{1}=0 \\
Z_{2}=20 \\
Z=\frac{Z_{1}}{Z_{2}}=0\end{array}$}} & $\Delta_{j a}$ & 16 & 30 & 21 & 0 & 0 & 0 & \\
\hline & & & & & $\Delta_{j b}$ & $58 / 3$ & 20 & $56 / 3$ & 0 & 0 & 0 & \\
\hline & & & & & $\Delta_{j}$ & 320 & 600 & 420 & 0 & 0 & 0 & \\
\hline
\end{tabular}

Solution 5.2: Solving the example 5.2 by new technique to modified simplex method and applied an algorithm in the section 4, after 2 steps we obtained the initial table as follows in table 5.2.1, after three iterations, we obtained the result in the following table 5.2.2.

Table 5.2.2: Final table for example 5.2 by new technique to modified simplex method

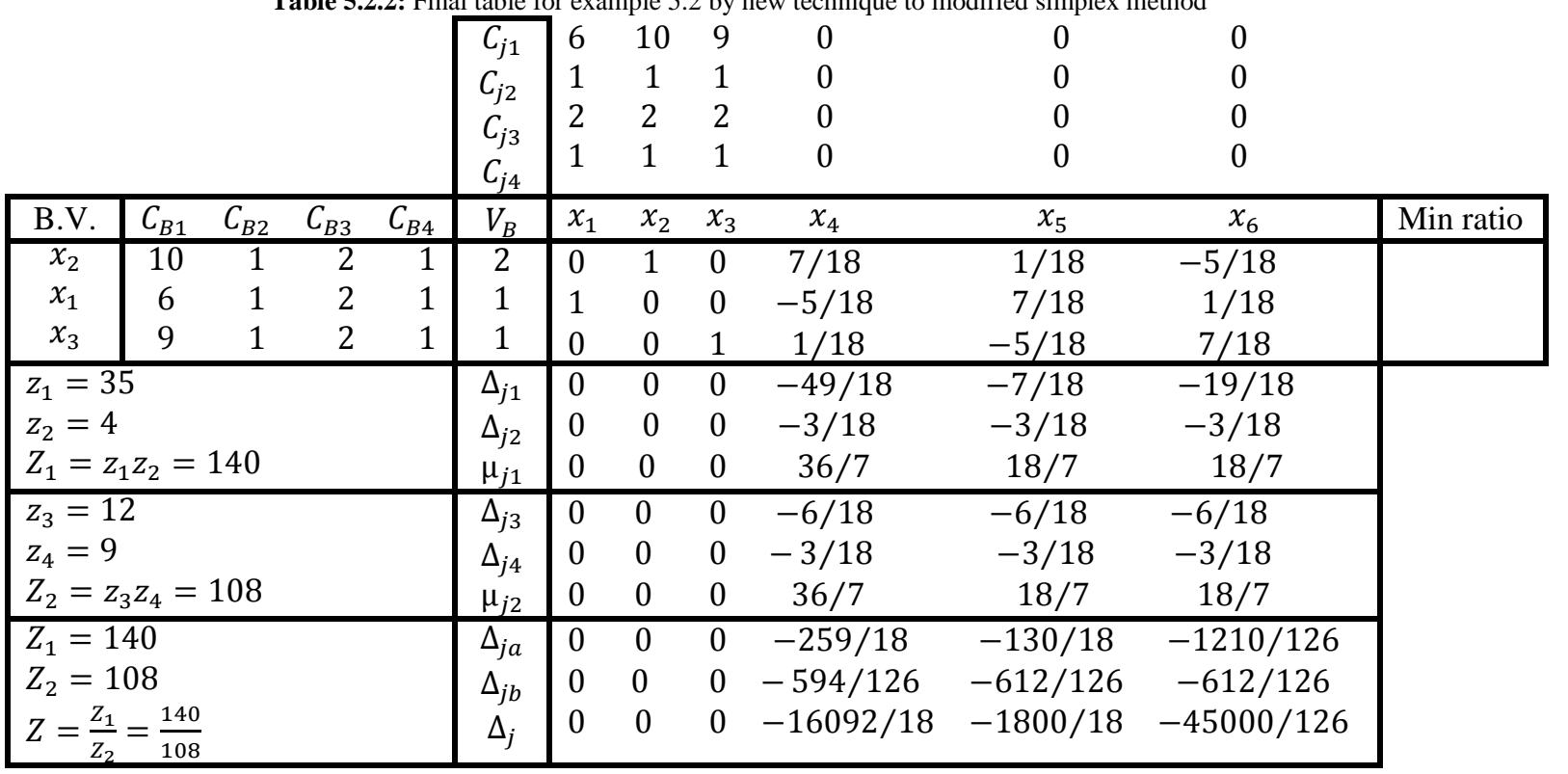


After solving it by new technique to modified simplex method, without complementary condition $\left(x_{2} x_{3}=0\right)$ we get Max. $Z=\frac{140}{108}$ and $x_{1}=1, x_{2}=2, x_{3}=1$

Now, we going to satisfied the complementary condition $\left(x_{2} x_{3}=0\right)$, because $x_{2} x_{3} \neq 0$. The optimal solution is $\operatorname{Max} . Z=\frac{78}{80}$ and $x_{1}=1, x_{2}=2, x_{3}=0$

\section{Discussion}

In this paper find CQFPP by the new technique to modified simplex method. The optimal solution must be at one of the complementary points of the polygon of the feasible region, sometimes it may be need to use technique for finding best complementary point solution for the problem.

\section{References}

[1] Arora, R. and Arora, S. R., "Solving Linear-Quadratic Bilevel Programming Problem Using Kuhn-Tucker Conditions", AMO-Advanced Modeling and Optimization, Vol. 13, No. 3, (2011), PP. 366-380, ISSN: 1841-4311.

[2] Arora, S. R. and Narang, R., "0-1 Bilevel Fractional Programming Problem with Independent Followers", International Journal of Optimization Theory, Methods and Applications, Global information Publisher (H.K), Co. , Ltd, Vol. 1, No. 2, (2009), PP. $225-238$.

[3] Fang, Shu-Cherng, Gao, D.Y., Sheu, Ruey-Lin, and Xing, W., "Global Optimization for a Class of Fractional Programming Problems", $J$ Glob Optim., Springer Science+ Business Media, LLC, Vol. 45, (2009), PP. 337-353.

[4] Gupta, A. K. and Sharma, J. K., "Quadratic Complementary Programming", Journal of the Korean Operations Research Society, Vol. 7 , (1982).

[5] Ibaraki, T., "Complementary Programming", Worlsing paper department of applied Mathematics and Statistics, Kyoto University, Kyoto, Japan, (1969).

[6] Ibaraki, T., "Complementary Programming", Operations Research, Vol. 19, No. 6, (1971), PP. 1523-1529.

[7] Jahan, S., and Islam, M. A., "A Complementary Slackness Theorem for Linear Fractional Programming Problem", International Journal of Basic and Applied Sciences IJBAS-IJENS, Vol. 10 , No. 2, (2010), PP. 39-44.

[8] Judice, J. J., "Algorithms for Linear Programming with Linear Complementarity Constraints", (2011).

[9] Sharma, S. D., "Operations Research", Kedar Nath Ram Nath BCO., Meerut, India, (1988), P(559).

[10] Sharma, S. D., "Nonlinear and Dynamic Programming", Kedar Nath Ram Nath and CO., Meerut, India, (1980), P(547).

[11] Sulaiman, N. A., "Extreme Point Quadratic Programming problem Techniques", M.Sc. thesis, University of Salahaddin, Hawler/Iraq, (1989). 